\title{
Systems logic for problem formulation and choice
}

\author{
E. L. Nappelbaum \\ Russian Institute for Systems Analysis \\ 9 Pr 60 Let Octabryja, Moscow 117312, Russia \\ Tel/Fax +7095282 4275 \\ e-mail: ernapp@aha.ru
}

\section{Abstract}

This paper provides a critique of the traditional practice of developing decision analysis and decision support techniques founded on a linear model of choice to frame the decision problem and its "solution".

Decision problem representation methods generally represent the problem in terms of some or all of the following five components: Alternatives or options, that is, different moulds in which the world around us will be cast as the result of our decision; the scope of these alternatives, which outlines the boundaries of the problem as conceived by the decision maker; the decision-makers' preferences, reflecting their attitudes and values as well as their understandings of the interests they have in the problem they are trying to tackle; the logic of choice, that is, the argumentation process through which we are capable of interrelating all the other components to arrive at the final decision; Instrumental intentions of the choice, combining both the reasons about how we are planning to exploit the results and the ideas of how choice is to be implemented.

In the conventional linear analysis, the first two components represent the "objective reality" of the choice, while the third (and to a certain extent the fourth) component introduce the subjective dimension of it. "objective reality" and subjective preferences are inputs to the choice procedure, which completes the analysis, and so instrumental intentions are often neglected.

Instead, a circular model of choice is proposed, in which the representation of all the above components can be balanced, and in which the knowledge and subjectivity of all the participants involved in the decision making and implementation process may be considered.

\section{Keywords}

Decision making, linear model of choice, circular model of choice, systems logic, problem formulation 


\section{INTRODUCTION}

If one chooses to look in a detached and objective manner at the field of science, often called cognitive or behavioural sciences, the latest attempts to study a variety of issues related to human action, creativity, problem-solving and decision-making (to mention just a few), one cannot fail but notice a certain trivialization of these issues. One can hardly avoid a feeling of uneasiness when one encounters the cases of equating rationality of choice with transitivity of preferences, equating learning with con-traction of the option set (either in the deterministic or in the probabilistic setting), equating goal-seeking behaviour with search of the optimal point (be it local or global) in a space of attributes, equating creativity with search in a maze and equating decision-making with folding of partial evaluations in a multi-attribute space. Other instances of the same kind are over abundant and can be found not only in papers which attempt some degree of formalization of the issues related to intelligent behaviour but also in works aimed at a more philosophical and comprehensive mastering of the subject. Perhaps, this is most vividly demonstrated in the field of artificial intelligence (AI) where most of the discussions, aimed originally at a deeper under-standing of major underlying issues, typically boil down to the exposition of some new scheme which is not up to all the intricacies of the arguments leading to its advent.

It is possibly even more surprising that, despite the uneasiness mentioned above, we time and again witness how these oversimplified generalisations lead to some important practical results and devices, to a better grasp of the same issues that have been construed in a rather caricatured way.

One of the possible explanations of this apparent paradox can be found in the general idea that the underlying principles of any purposeful (or, at least, any cognitive) behaviour should be very simple as if by definition and that it is only the complexity of the environment over which this behaviour unfolds itself that makes the outward manifestations of this behaviour so intricate and messy. Such a stance has been argued quite persuasively by $\mathrm{H}$. Simon $(1967,1969)$ among others, and is the core of some recent theorising (e.g., the mutual-causality theory of Maruyama, 1963). Nevertheless, the uneasy feeling mentioned above does not vanish and, indeed, it comes to grow even stronger with each attempt to extend the scope of application of some particular explanatory scheme which was locally proven to be quite successful.

The situation reminds one quite distinctly of the early 'untransferability" of the first tools of artificial intelligence where each new successful solution had a very strong ad-hoc flavour and did not lend itself to easy generalisation and dissemination as a general instrument of tacking Al problems (see, for example, Boden, 1977). Moreover, sometimes the mere possibility of explaining complex behaviour on the basis of a relatively simple generative rule appears to be insufficient for admitting 
it as the explanation. Furthermore, external compatibility of the explanation with observed behaviour by no means guarantees the validity of the explanation. ${ }^{\prime}$

In this paper, I would like to go beyond merely expressing the afore mentioned doubts and argue that, whenever something can be explained within a framework based on relatively simple underlying principles, it would not be a very gross exaggeration to state that the particular mechanics of the scheme are nearly irrelevant and that there may exist a number of similar explanatory schemes that will do nearly equally well. In other words, I would like to argue that, in each explanatory scheme used to analyse intelligent behaviour and, indeed, to design a more intelligent behaviour, there is always more than what meets the eye and what is put generally forward as the main vehicle of the logic of the analysis and design. The following specific examples may help clarify this claim.

One of the most developed area of Al is pattern recognition (in fact, it is so well developed and advanced that recently we have started actually to regard it as a field apart). A lot of effort has been spent in this area to refine various algorithms for classification, discrimination and learning based on various underlying assumptions. In their practical applications, however, techniques employing such algorithms all score either almost equally well or not at all. This statement, of course, is not entirely accurate as some problems will always exist which adapt especially well to the language of one specific approach (especially if the languages of the problem descriptions are structured dissimilarly) and it is precisely those problems that the authors of a particular technique refer to when they wish to demonstrate the superiority of their brainchild. In most of the other cases, however, it has proved nearly always possible to reformulate the original problem in such terms that, within the given pattern representation, a different technique will perform nearly as well as the original one.

In the light of such evidence, it is quite easy to start musing about whether it is really the implementation technique to which the success has to be attributed rather than to the chosen language of the pattern representation. By the same token, we cannot fail to notice that the vast variety of econometric models used to predict the next year's GNP and the like fare nearly equally well, independently of the many differences in their underlying assumptions and independently of the particular choice of the logic of extrapolation. At the same time, all of these econometric models fail rather dismally whenever some structural change happens in reality calling for a parallel change in the structure of their source data. Finally, the entire history of decision-making methodology has proven times and again that, whenever we have found an appropriate language to represent our options, nearly any reasonable scheme of analysis of multi-attribute decisions will do and that, in the absence of the proper understanding of the real content of the options, no such

\footnotetext{
' Some really deep analysis of the difficulties which may be encountered along this way can be found in Humphreys and Berkeley (1985).
} 
device will be of any help. Given this brief discussion, I am quite sure that the reader will have no difficulty in providing some more examples on his own.

In the suspicions formulated above, I am ready to claim that, whenever a problem that requires a lot of thinking and creativity, when it is dealt with in an ordinary manner, and when it can be tackled by a relatively simple means within a theoretical framework, the success of its solution should be attributed mainly to a fortunate and judicious way of representing the problem rather than to the formal logic of manipulation of this representation.

\section{REPRESENTATIONS AND THE LOGIC OF CHOICE}

There is nothing new, of course, in this highlighting of the crucial importance of adequate representation. Recently, the problem of representation has been constantly in the very focus of scientific attention (see e.g. Bobrow and Collins, 1968; Fodor, 1981, Schank, 1986) and many interesting and valuable ideas have come to their fruition in this way. ${ }^{2}$ The representation problem, however, is dealt with as if $i t$ were a separate issue, as if it were actually possible to look first for an adequate representation, and only after that, choose one to try finding an appropriate way of solving the problem thus represented. One of the main contentions of the present paper is that this kind of decomposition of problem solving activity is possibly a major source of trivialization and that, in reality (that is, in really creative thinking and problem-solving), the process of choosing the proper representation just can not be divorced from both problem formulation and problem solving.

In order to argue this viewpoint I would like to consider first our basic understanding of the logic of choice, the possibility of its application to the selection of an adequate representation for a problem and its inner relation with the problems of cognition on the basis of the general ideas suggested in Emelyanov and Nappelbaum (1985). Independent of the level of our contemplation of a decision problem, be it purely theoretical or imminently practical, and independent of what particular kind of thought we are trying to follow in organising these thoughts, be it the logic suggested by Jeffrey (1965), or the methodology put forward by white (1975), or the ideas of Simon (1960), we always represent the problem in terms of the following four major components:

\footnotetext{
${ }^{2}$ Nonetheless, we can notice some traces of trivialization in this particular area of study as well. For instance, the formalisms of the frames which are currently in existence appear to be much more limited, restrictive and impoverished in comparison to the general considerations used to erect the foundation for them (see Minsky, 1975).
} 
- Alternatives or options: that is, different moulds in which the world around us will be cast as the result of our decision;

- The scope of these alternatives, which outlines the boundaries of the problem as conceived by the decision-maker;

- The decision-makers' preferences reflecting their attitudes and values as well as their understandings of the interests they have in the problem they are trying to tackle;

- The logic of choice, that is, the argumentation process through which we are capable of interrelating all the other components to arrive at the final decision (i.e., to choose the preferred alternative).

The first two of these components represent, as it were, the objective reality of the choice while the third and, to a certain extent, the fourth introduce the subjective dimension of it (see Figure 1). Indeed, it is in the first two components in which all the information about the objective possibilities and the objective constraints relevant to the problem under consideration are embodied while the third component introduces subjective personal attitudes towards various facets of the different worlds which should emerge as a result of the choice. As to the logic of choice it embodies, this comes essentially from the methodology of traditional science where it prevails. Indeed, the logic of choice, as depicted in Figure 1, independently of the algorithm of evaluation and of folding of partial criteria, can be interpreted as a result of a conjoint measurement (Krantz et al, 1971) of the utility of options performed by humans seen as instruments. An equally valid alternative interpretation explicates options as instruments, while the choice becomes a conjoint measurement of the attitudes of humans. This latter interpretation is especially useful in the context of revealed preference analysis.

In this sense, the only, rather limited, place for creative reasoning in the choice process is in the folding of partial criteria and in finding appropriate trade-offs between gains and losses achieved on various dimensions of the option characterisation. And there is nothing accidental in the fact that such a profound thinker as Simon (1969) was quite adamant at stating that there is no special need for a separate logic of choice as it is patently sufficient to analyse the consequences of various choices and to select those which seem to be more appealing.

The situation, however, is by no means that simple. First of all, the first three components of the problem characterisation are mutually inter-dependent. Indeed, the options which we see fit to consider are not just names or labels but rather manifestly incomplete representations of the reality, bundles of attributes which we consider to be essential for ultimate evaluation, and, as such, the choice of a representation for the options depends on the choice of the preferences. Conversely, with different representations of the options, we perceive these options differently, and, consequently, apply different criteria in their comparison.

At first glance, it may seem that the scope of alternatives to consider is independent of both the choice of representation and the preferences. Meanwhile, it is only 
rarely that this set of alternatives turns out to be the result of a formal combinatorics in the attribute space and, indeed, examination of the rejected combinations quite often reveals semantic relations which only rarely find their proper expression in the representations chosen. ${ }^{3}$ Moreover, it seems patently unsatisfactory to reduce the reasoning about one's choice to a mere folding of partial evaluations of alternatives in terms of partial preferences seen as projections of some "global preference" to be revealed (see Emelyanov and Nappelbaum, 1977). In order to demonstrate this to one's own satisfaction it is sufficient to try to explain in these terms the human capability of changing one's opinion, or reframing, or the phenomena of external persuasion. Indeed, it seems to be extremely difficult to explain all these discontinuities in the process of reasoning, and all these instances of illumination and revelation in terms of gradual updating of the parameters of the criterion folding mechanisms. It is even more difficult to imagine in what terms one may argue about the nature of the mechanism itself. ${ }^{4}$

Some argue, of course, that the formulation of the choice problem is always preceded by its holistic perception and this is a Gestalt perception (Mandler and Mandler, 1964) which is focusing our attention to the relevant representation and a proper choice of preferences. ${ }^{5}$ This brings us to consider another, fifth, component of the choice problem formulation which is only too often misguidedly neglected when contemplating a choice situation:

- Instrumental intentions of the choice, combining both the reasons about how we are planning to exploit the results of the choice and the ideas of how the choice is to be implemented.

One of the first attempts to attract our attention to this aspect of the comprehension of the choice situation can be found in Lancaster (1966). The need for such a component becomes quite clear if we return to the original intention of the paper which is to reason about the choice of representation. Indeed, it would be difficult even to start thinking about a proper way of representing something before having a rather clear idea about what we are going to do with this representation, both

\footnotetext{
${ }^{3}$ The information gained through such an examination is, actually, capitalised upon in many creative techniques such as morphology analysis Koberg and Bagnail, 1974) or synectics (Cordon, 1961).

${ }^{4}$ It is interesting to note that though it is precisely this objective which is claimed in the title of the book by van Grich (1987), the content of the hook attacks very different and much more interesting topics.
}
${ }^{5}$ A more detailed analysis of such a shift in the focus of our attention with regard to a problem of choice can be found in Humphreys and Berkeley (1983). 
meaningfully and formally. Moreover, it is precisely this choice of intentions that relates the problem of choice of the representation to the problem of choice of a substantive alternative.

In addition, I would like to draw the reader's attention to the diversity of various problems of choice (of representation, of preferences, of the logic of choice, of intentions, of the set of alternatives etc.) we are forced to consider when we attempt to analyse the finer points of the choice procedure.

Elsewhere Emelyanov and Nappelbaum, 1985)1 have argued that a natural way of avoiding the tenets of interdependencies of the various components mentioned above (as well as the infinite replicative proliferation of the choice problems of both lower and higher levels) is to abandon completely the implicative linear logic underlying the approach shown in Figure 1 and to start analysing the problem in the light of an entirely different "circular" logic based on the ideas of balance.

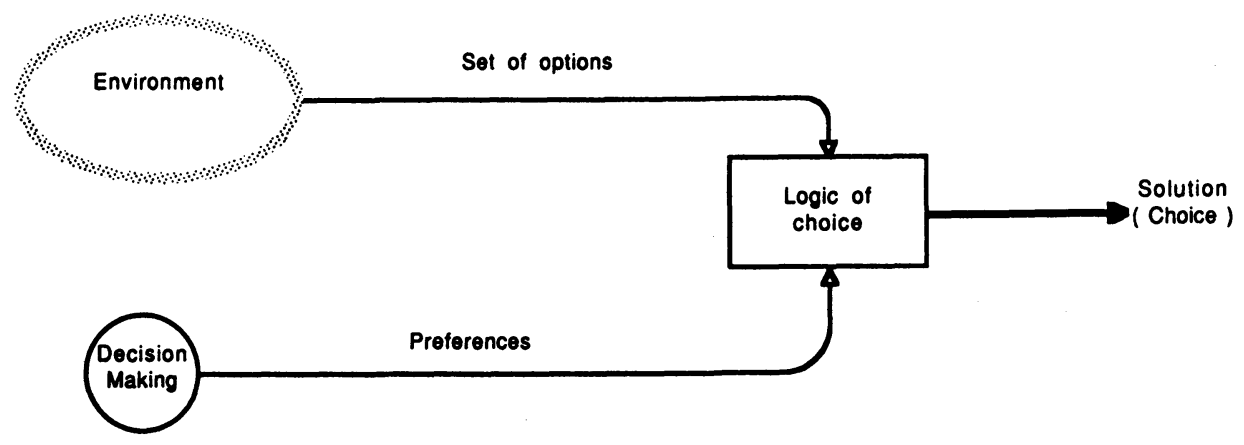

Figure 1 The linear logic of choice

The idea of balance as an underlying principle governing human behaviour is by no means new either in psychology (Heider, 1946) or in cybernetics (Pask, 1975). It was strongly advocated by the proponents of cognitive dissonance (Festinger, 1957) though this school of thought has failed, in my opinion, to bring it to its natural conclusions through trying to temper it with the ideas of maximisation which is foreign to the principle of balance (something is either balanced or not and so it is rather strange to speak of the degree to which it is balanced).

Within the general framework I propose here, the idea of utility maximisation is rejected totally as a foundation of choice or, for that matter, of any form of intelligent purposeful behaviour. Within this framework, in the process of choice, we do not choose only an option but, rather, choose, concurrently and interdependently, all the five components of the problem formulation outlined above and select the option which in the light of this formulation we will consider as the choice. In doing so, we shift our attention from the problems of utility 
measurement to the problems of construction of the languages in which we are going to describe our options (as well as discriminate between them), the values (or preferences) we see as relevant, and our intentions of actions (both for implementation and utilisation) as well as the set of alternatives to consider and the logic of choice to apply. I have tried to capture this change of the logic of choice in graphical terms through changing from the scheme of Figure 1 to that of Figure 2.

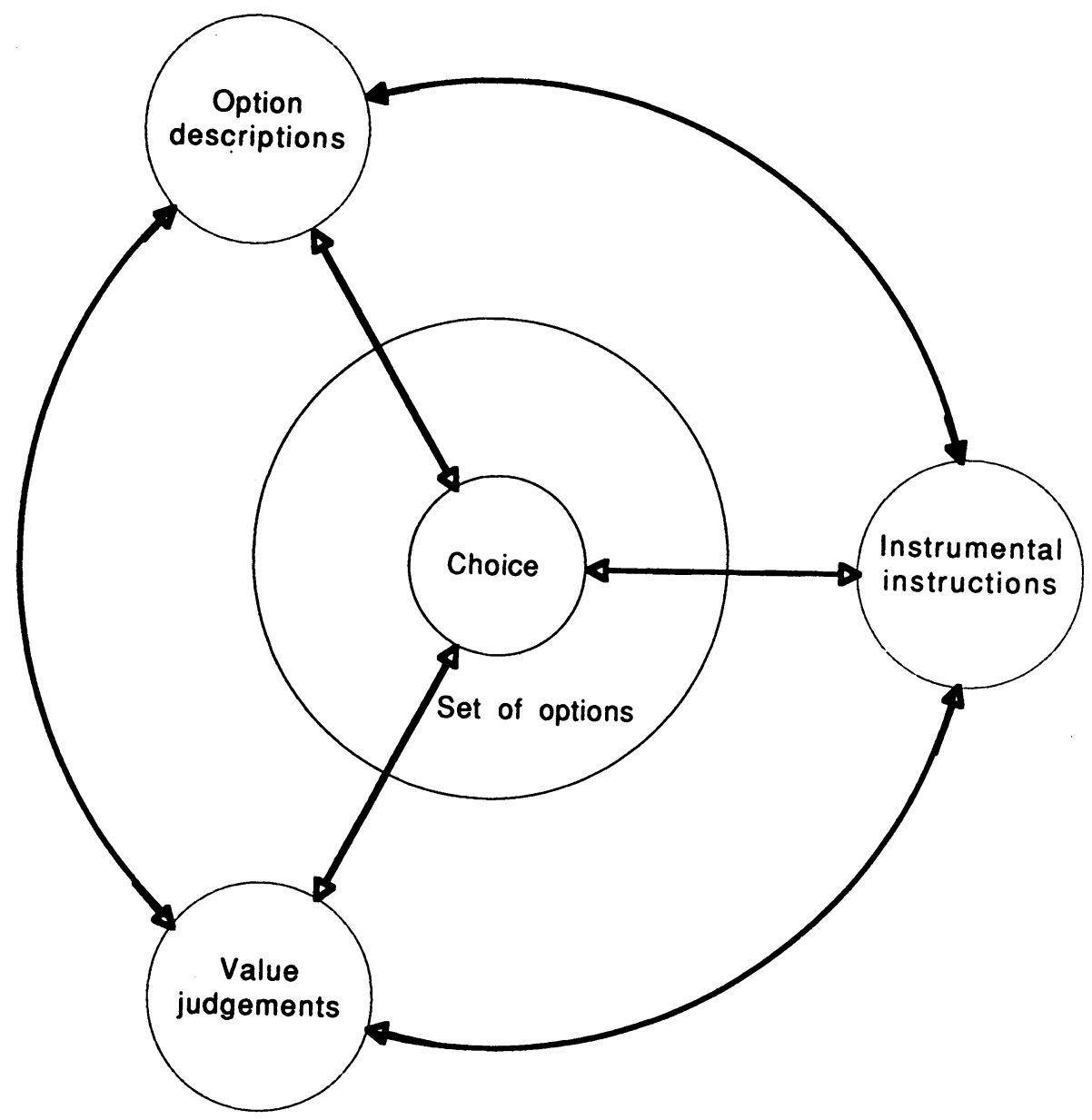

Figure 2 The circular logic of choice

The scheme sketched in Figure 2 places a heavy emphasis on the dynamics of construction of various representations or languages (very much in the sense of Pask's, 1975, conversation theory) which proceeds until we reach the balance consisting in an obvious self-sufficiency of data (that is, when the data conveyed by a representation of each particular kind shown in a circle in Figure 2 are both 
sufficient and necessary for applying representations of the remaining kinds) and in an inner consistency which requires that the original dilemma of choice disappears altogether as, under the constructed representation, one of the options now dominates all the rest quite clearly.

In actual terms this means that we look for or, rather, design such an overall representation of the choice situation (with declarative, instrumental and the value components as well as with the choice boundary indicated) that makes the problem of choice disappear altogether in the sense of removing completely all the doubts (or cognitive dissonance) produced by the previous embaras du choix. The problem of choice, therefore, turns out to be reduced to the problem of problem-solving, that is, to the problem of finding a representation which balances out in the sense explained above. Such a change of the general perspective leads to several important conclusions about how we deal with the problems of choice in general.

First of all, due to the need for inner consistency, the resulting (holistic) representation should be, by necessity, a very compact one. A more complicated version of representation would introduce unavoidable cognitive dissonance, contradictory evaluations such that the representation loses its holistic, Gestalt nature.

Even with the simplest kind of representation it is not always possible to achieve the balance we seek. Hence, many a time the balance is reached through liberal use of "default" elements of representation for which the true information is either lacking or substituted for by something we wistfully wish to happen even against the strongest evidence.

The suggested compactness of the holistic representation along with its balanced nature makes it quite easy to mould the inner reasoning it is built around into a variety of seemingly different forms of implicative deductive kind. Hence, there exists a variety of different soft methods of systems analysis (Checkland, 1981) or, by the same token, of methods dealing with decisionmaking, forecasting, knowledge integration etc. In reality, however, they are all just a rationalisation of an ad hoc circular representation logic ("successful" or otherwise).

The latter consideration can provide an explanation of the seeming simplicity of intelligent behaviour as well as of the trivialization of some crucial and profound notions. As soon as we have been really successful in capturing the essence of a situation (as expressed by our capability to design a holistic representation of it), we can mould the underlying reasoning within a simple framework, while some of the features of this logic (such as transitivity of preferences, independence of attributes, minimisation of the descriptive space, etc.). Any attempt to characterise this intelligence, rationality or creativity in these terms thus merely reflects our intention of working within this logic rather than really uncovering some property of the intellect, of rationality or of creativity. 
Because of the compactness dictated by the inner consistency principle, the balance achieved is rather fragile and temporal but, at the same time, it is essentially attribute-tight and isolated in the sense that has been brought to our attention by the ideas of autopoiesis (Maturana and Varela, 1979). But, by the same token, this implies that, for the same actual situation, it is always possible to design several competing representations. Thus, the depth of our comprehension of the situation depends crucially on the number of representations one may design for it, rather than on the comprehensiveness of any of these representations (this latter criterion is self-contradictory in any case).

These representations are conceptually discrete in the sense that one cannot move from one to the other in an evolutionary manner (e.g., by modifying gradually the weight one may place on an attribute). They are also conceptually incomparable because they exist in different psychological worlds where even the same variables may have different interpretations. Any simple merger of these worlds is impossible precisely because of the organisationally closed semantic and pragmatic constriction of the balanced representation which constitutes each of them.

This cognitive closure not only involves the dimensions that enter explicitly in the representation but also explicitly presupposes a certain understanding (or a cognitive commitment) that all the remaining dimensions do not threaten the integrity of this closure. Any change of the situation may, in principle, invalidate this assumption and, hence, such a representation may be a very poor candidate for transitive chaining. This may explain the well-known phenomenon whereby an originally quite interesting and meaningful statement of a problem may become quite artificial and even nonsensical with further attempts at its generalisation and development.

The possibility of having several incomparable and equally valid representations of the same actual situation calls for considering the problem of choice at another level, that is, of choosing one of these representations (small worlds). As I have pointed out earlier, this problem of choosing the appropriate representation cannot be solved through a mere merger of all the relevant representations.

This new problem should be treated exactly in the same terms in which we tackled the original one: we have to design for it an entirely different representation, with its own options, its own declarative language, its own higher-level values, and its instrumental intentions (see Figure 3 for a schematic explication of the interlevel logic dependencies). As such, it addresses a completely different scope of reality with the languages designed for the original choice being singularly inappropriate for this new level of contemplation.

At first glance, it may seem that these last propositions bring us firmly back to the difficulties related to the phenomena of proliferation of problems of choice. The situation, however, is not equivalent to that of Figure 1. Indeed, to be able to create a higher level of the choice uncertainty, one has to design several representations of the basis of choice between several viewpoints embodied in the separate 
representations of the original level. This, however, calls for additional efforts and the margin left by previous decisions which have committed us to some higherlevel logic may be rather limited.

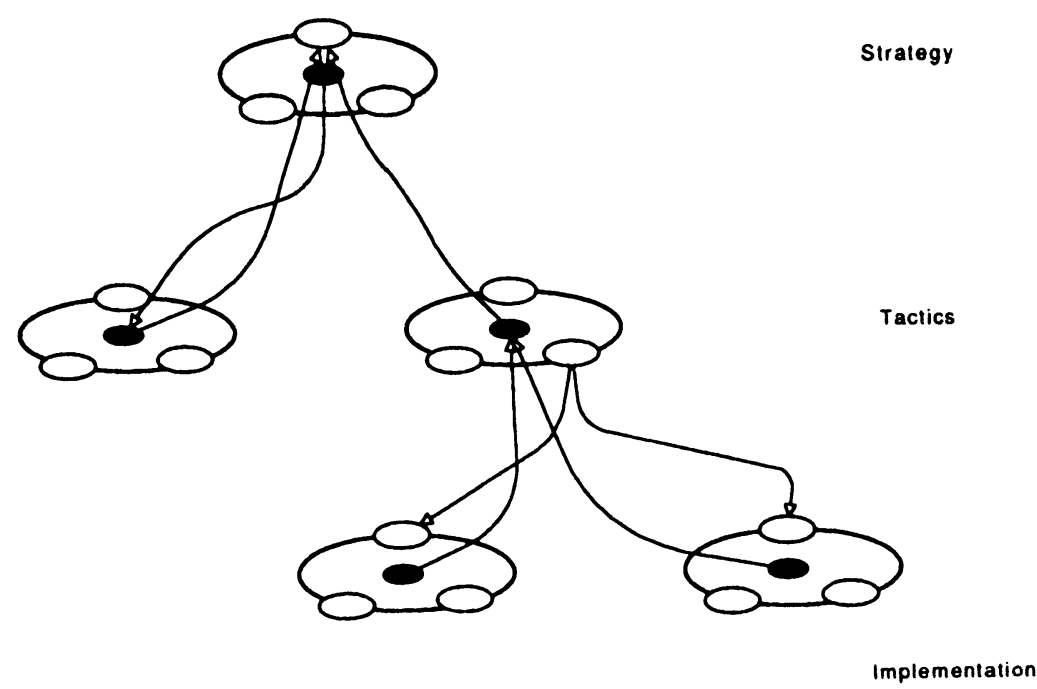

Figure 3 Interlevel dependencies in the circular logic of choice.

Another restricting factor may be associated with the reasons of implementation of the chosen option. The shift of attention to various individual actions involved in implementation must be followed by re-focusing our perception of the situation, by the design of a new representation in terms of which something which originally looked to be the solution may now appear not to be so.

Similar considerations of the economics of design of holistic representations make us understand some aspects of the dual role played by stereotyping in cognition and thinking. On the one hand, stereotyping is a highly useful device helping us to economise on the representation design and to organise incoming information in a more efficient fashion (this aspect has been discussed elsewhere, e.g. in Pospelov, 1981, with respect to the frame ideas). But, on the other hand, it makes our thinking less flexible and less open to new ideas, it creates conceptual blocks to be discarded, and it makes us blind not only to some (possibly crucial) details which fall outside the stereotyped perception, but also to some fundamental assumptions underlying the semantic framework of the stereotype (Mason and Mitroff, 1981). The same considerations may give us some answers to the questions formulated in the beginning of this paper. Indeed, it is the design of a 
new balanced representation, and thus the achievement of a new cognitive closure (be it self-made or suggested by somebody else) that may lead us to a change of mind, to reframing.

\section{SYSTEMS ANALYSIS AND ORGANIZATION OF KNOWLEDGE}

An attentive reader may have possibly noticed that, though I have started to discuss the logic of choice, my last comments apply equally well (and, indeed, were intended to apply) in more general settings. This is fully in line with my general contention that, actually, any problem of choice reduces itself, in the final analysis, to a problem-solving kind, while the solution of any problem of the latter type depends crucially on the appropriate choice of the problem representation.

In problem-solving terms, Figure 2 can be interpreted (see Figure 4) as a representation of the logic of problem formulation with declarative (description of the problem search space), instrumental (description of the operators or transformations in the problem search space), and value (definition of a solution or of an instrument for identifying one) components which must be self-sufficient and properly balanced (in the sense that they suggest a solution) since, in the final analysis, only the existence of a solution makes the problem formulation appropriate.

Figure 4 The circular logic of problem formulation

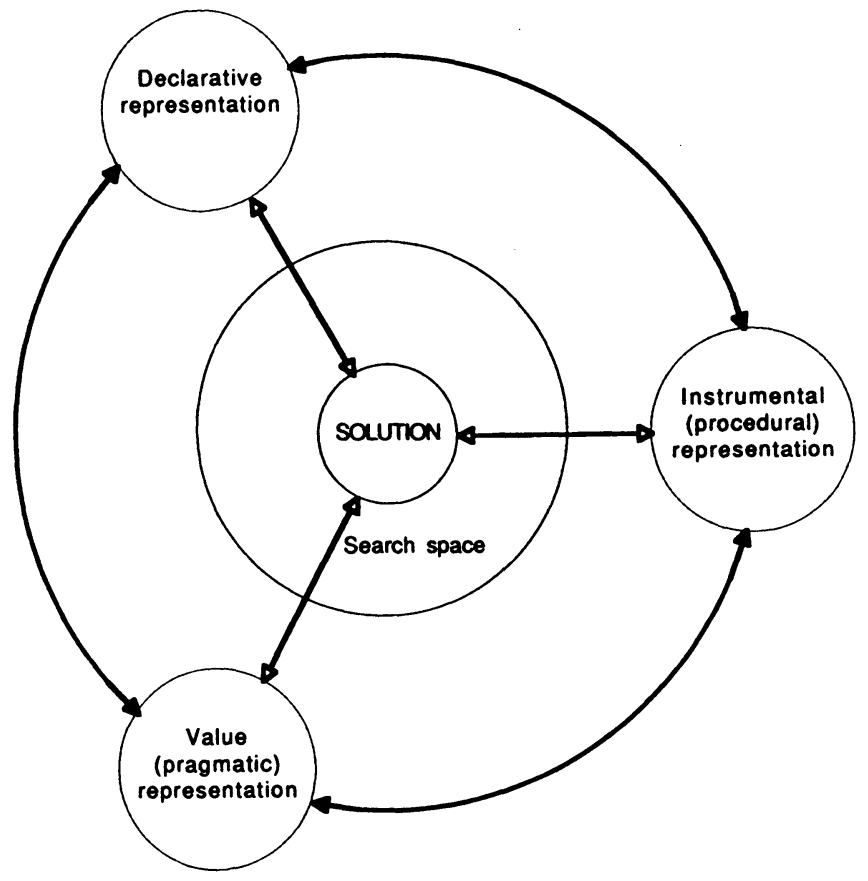


In this sense, there is actually none of the customary time distance that separates the formulation of the problem from the instance of arriving at its solution. The problem is not really formulated (at least, we cannot be sure of it) till it is solved and vice versa. The validity of this statement is rather evident when we deal with what are called soft or ill-structured problems but it is also equally true in much more rigorous, well-structured settings. As Lakatos (1963) has brilliantly demonstrated, even in the limiting case of a mathematical problem, we always start with a premonition of a specific solution; we know it, though possibly mistakenly, and only after that, we start to argue it or rationalise it. of course, in the course of the latter process, we may well find a need for changing the solution and for qualifying or modifying the original formulation and for rejecting either or both of them.

In reporting the problem solution, however, we rarely (if at all) describe or even refer to the process through which we originally arrived at the solution. Moreover, we present it as if the solution were originally divorced from the problem formulation and has come to us as a total surprise. Note that in the case of the most well-structured type of knowledge, i.e. in mathematics, it is customary to present the problem statement and the problem solution simultaneously and on the same footing in the form of a theorem. ${ }^{6}$

All the processes related to the formulation of a problem and to the simultaneous discovery of a solution, were for a very long time thought to be outside the realm of science. It is only recently that we have started to consider these processes as subject-matter of scientific research, of professional skill acquisition (e.g., within Operations Research groups, independent consulting etc.), and of special-purpose techniques and devices. They have come to be an important part of the systems analysis methodology (the chronology of development along these lines are well covered in Checkland, 1981). They are also quite prominent in various creativity enhancement techniques (de Bono, 1970; Adams, 1979), and they lie at the core of many $\mathrm{Al}$ research projects, though in a heavily camouflaged form.

This burst of interest is not so difficult to understand. The increased pace of development as well as the higher complexity of the processes we have to deal with have prompted a strong demand for speedy problem-solving to cope with the realities of modern times. While quite a few of these problems are standard, at least from the structural viewpoint (hence the emergence of Operations Research as a problem solving discipline), many of these problems are in principle unique, welladapted only to the current environment and have to be handled without delay, thus

${ }^{6}$ The same standard does not, however, apply to the companion problem of finding the proof. Here, both the problem formulation and the way of arriving at the proof are left hidden from the audience. 
allowing little time for an illuminating insight to produce itself from an unstructured contemplation of the problematique.

Though to-day we know of several competing standard techniques or even methodologies for problem formulation or conceptualisation, most of them do not do real justice to the multi-faceted nature of the process. The majority start by considering a particular, strictly defined, component of the problem representation and try to unfold it into a fully-fledged conceptualisation in a kind of implicative fashion. True, many if not all of them provide for some feedback but this is only a poor relation of the circular balancing logic we wish to advocate. At the stage where feedback is involved, the conceptualisation will already have gone sufficiently far to prefocus our attention on a particular framework, and so the feedback comes in useful unly as an instrument of fine tuning rather than for reconceptualization of the problem.

Within the framework proposed here, it is essential to see that there are many entry points into the process of conceptualisation, and none of them may claim the privileged role. We may enter the problem from a prospective declarative representation (i.e., by initially choosing the description of the space where we shall develop our options), or from an analysis of the instrumental possibilities we have (i.e., from a set of actions we see as relevant and feasible with respect to the problematique), or from the angle of the value components (i.e., by stating our objectives and goals), or from a detailed analysis of a particular option we have in mind (e.g., by asking ourselves why we are dissatisfied with the status quo), or, indeed, from a detailed analysis of the scope of the options, leading to the better understanding of hidden assumptions manifest in implicit constraints. And, of course, the actual entry point may be a combination of those mentioned above.

Independently of the entry point we have chosen, however, it is essential that our cognitive logic of conceptualisation is inherently flexible enough not to keep us within the same frame that was premeditated by the entry representation. Choice of a particular entry point should not mean that we are doomed to stay forever within its domain. It is precisely in this feature that many of the current conceptualisation techniques are lacking.

What we really need is a comprehensive battery of methodologies and techniques that will allow us to analyse the inherent structure of an existing stereotype, to reveal the hidden assumptions which underly it, to burst and disintegrate it and to use the debris as entry points into entirely new creative cognitive closures. To be able to achieve all this we need, of course, an underlying theory and a set of appropriate meta-principles. In other words, we need a developed understanding of the principles of knowledge organisation that make it possible for human beings to solve problems efficiently and creatively, to make decisions and, in general, to interact intelligently with the world in which they live. In my view, it is precisely this objective that systems analysis and design, operations research, decisionmaking methodology, design methodology and many other similar fields are trying 
to achieve, though this idea is present in most of these fields in an implicit and subconscious form.

Systems research, it seems, has achieved a closer grasp of the topic than have any of the others as it is much more flexible in its choice of entry points and adopts fewer assumptions which make some components of original formulation of the problem unalterable. We also hope that the general scheme of organisation of knowledge into an integrated atomic whole presented earlier may serve as a foundation of this new science of the knowledge organisation into system is understood to be specific forms of knowledge organisation especially suitable for intelligent interaction with the environment (Emelyanov and Nappelbaum, 1985).

In order to be in line with our own conception we have to accept the possibility of another organisation of knowledge that constitutes the entire problem domain of systems research. Indeed, in a sense, this paper is trying to present a cognitive closure for development of systems methodology and methods with the current dissatisfaction with their explication taken as the problematique which we are quite aware but do not really know what to do with. The only feature that puts our thesis apart is that it is self-contained, that is, the whole approach can be derived from the same principles it applies to the analysis of situations within systems practice and systems theory.

Moreover, many of the principles that have been proclaimed by many other explications only as imperative desiderata, as some meta-axioms, come here as natural conclusions. Some of these have been considered elsewhere (e.g. in Emelyanov and Nappelbaum, 1985). Here I would like to dwell a bit on a general belief that systems analysis offers a kit of tools especially suited for handling new kinds of problems: those which are described as ill-structured.

Although I fully sympathise with many intentions and assumptions that lie behind this claim, I am afraid that its actual wording is rather unfortunate and may be rather misguiding. First of all, by contrasting ill-structured and well-structured problems we are hiding the fact that in every human activity, however wellstructured it may be, we always start with an extremely ill-structured problem and always end up by solving a well-structured problem derived from and substituted for the original ill-structured one. But in order to be able to substantiate this contention we first have to consider what kind of a structure we have in mind when we speak about ill- or well-structured problems.

Generally, ill-structuredness is seen in terms of the inherent fallibility of the original formulation of the problem which reflects a high level of cognitive complexity of the environment we are trying to cope with. Ackoff and Emery (1974) speak of the messiness of such problems. Pospelov and Pushkin (1975) point to the differences between the language of description of the world we deal with and the language in which we describe our objectives and our goals. Larichev (1979) sees the problem as a patchy, partial way of describing our criteria of comparison. Mason and Mitroff (1981) give a comprehensive list of weaknesses and stress that, in an ill-structured problem, not only there is no means of 
distinguishing a solution but also there is no hope of achieving one that will remove the problem altogether. Within the framework of this paper, all this can be seen as an original imbalance of the problem presentation to be used as issue for ultimate removal from the problem formulation. This is, however, only partially true.

It seems to be true that in every ill-structured problem the main criterion of balancing (i.e., that of internal consistency) is not met. The original formulation either points to a variety of apparent solutions which we do not know how to distinguish between or apparently offers no guidelines for searching for a solution. In a sense, both of these cases amount to the same thing: the impossibility to implement something which we would see as a solution.

Indeed, even the most blatantly ill-structured problem (e.g., "I am quite unhappy about the present state of the things and I have to do something to improve it") is actually perfectly balanced. It is obviously self-sufficient and internally consistent as it points to an obvious and immediate solution: "Do something efficient". The problem, however, is that this "solution" does not tell us anything about how we can implement it. And, as soon as we try to get some better understanding of the state of things and of the ways to improve it (i.e., as soon as we start asking ourselves what we are dissatisfied with, what we can do, how we will distinguish an improvement etc.), we will also need to change all the other components of the problem statement to preserve the balance which was so easily achieved originally.

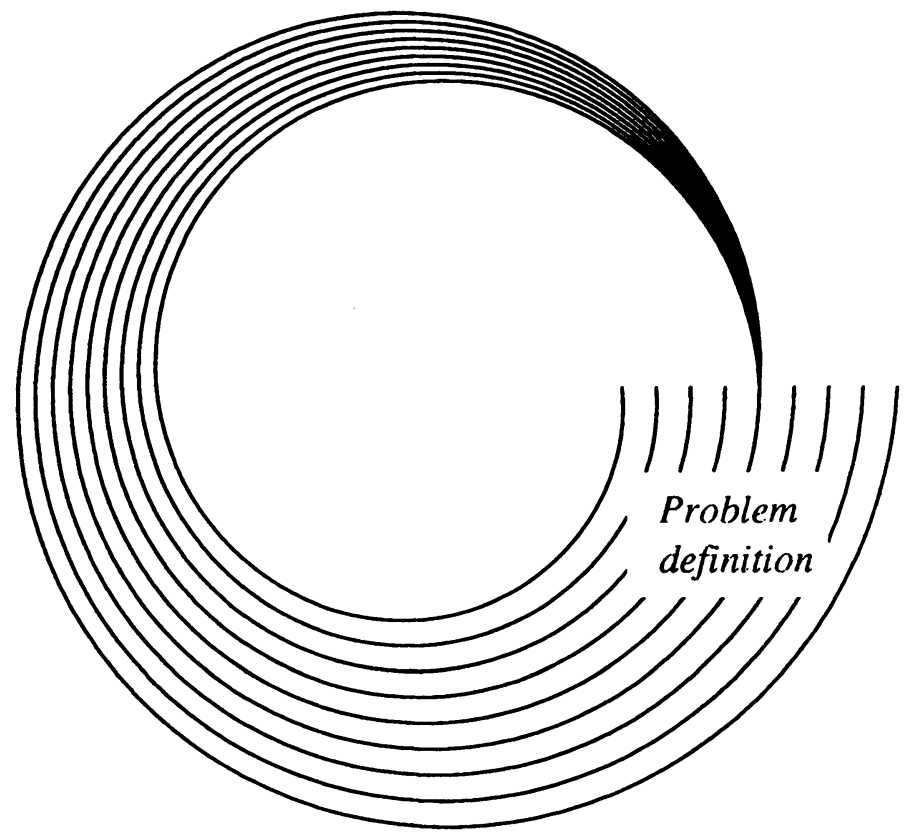

Figure 5 The problem definition cycle 
In reality, this means that we are forced to reformat our problem in a way that will make it narrower, more pointed, till an implementable solution emerges as the sole natural consequence of the new formulation. I have tried to express this idea graphically in Figure 5 which focuses on the fact that, as a result of a Systems analysis of a problem, we return to the same stage from which we have started, that is, to the problem formulation, though now this is sharpened by what we have gained in the course of our analysis.

Unfortunately, however, only in a few cases are we likely to do this by invoking some meaningful consideration. More often than not we do it by an implicit (and sometimes even unrecognised) substitution of the original problem by another one obtained either through an arbitrary prefocusing on some "obvious" way of dealing with the problem, or through the introduction of some "metacriteria" of rationality imposing rather arbitrary constraints on possible solutions ${ }^{7}$

In reality, all these meta-considerations are not actually the inherent features of a solution but, rather, result from the problem analyst's desire to make his or her life a bit easier. Their adoption in any practical case should be conditional on checking that the assumptions which underly their construction are met in the case in question. In many cases however this is not done as they are not considered by the problem analyst to be important enough to be verified regardless of the cost incurred in meeting them. As a result, intransitivities detected within the problem representation constrained by the meta criteria may be viewed as originating through "subjective error" in developing the problem representation. This ignores their real value as pointers towards aspects of the situation which control the problem definition, which are not representable within the current constrained representation, but which should play an important part in a representation which was closed in a more appropriate way (Berkeley and Humphreys, 1982).

In terms of the framework presented in this paper, such unnatural ways of narrowing the problem statement constitutes just another instance of default reasoning in achieving a balanced representation of the second-order problem, i.e., of designing a balanced representation of the original meaningful problem. In this respect, it would be useful to recall that, within the framework we discuss here, the ultimate choice of a representation (and hence of a solution) depends very much on implicit constraints placed both from above by the considerations of a higher "ethical" level, and from below by considerations of implementability of the instrumental intentions proclaimed in the representation. In a sense, all this has to

\footnotetext{
${ }^{7}$ The whole philosophy of optimality is a vivid demonstration of such an approach and it had a telling effect on many fields of research that may possibly never have used the word "optimality" in their professional language; the same comments apply to the ideas of transitivity or independence in the choice theory etc.
} 
be considered concurrently with the present level" representations and, in this sense, the diagram in Figure 6 is more appropriate than that in Figure 5.

Because of the limitations on the size of this paper it is not possible to develop this last ideas any further here. I will note only that, in line with the framework of Figure 6, the principles of balancing should encompass not only a single holistic representation but rather the entire branching chain of those stressing once again the inseparability of analytical, design and implementation components of a problem.

This brings also to the fore the temporal aspect of the problem designing exercise. First of all, the greater the number of levels we are trying to include in our analysis/design problem solving the greater the time scope of our analysis both in terms of the time required to solve the problem and the time horizon of the designed scenario. Meanwhile, we should be equally aware of the inherently provisional nature of the balance we have managed to achieve. Therefore, it is always necessary to look for a proper trade-off between the depth of the analysis and the temporary nature of the framework one has adopted for this particular purpose, that is, to find the borderline between determination and obstinacy. The system of values we adopt within this framework, and redesign again and again, is the most powerful tool we have for coping with this problem. Most of the heuristics considered in Newell and Simon (1972). can be explained in these terms as well.

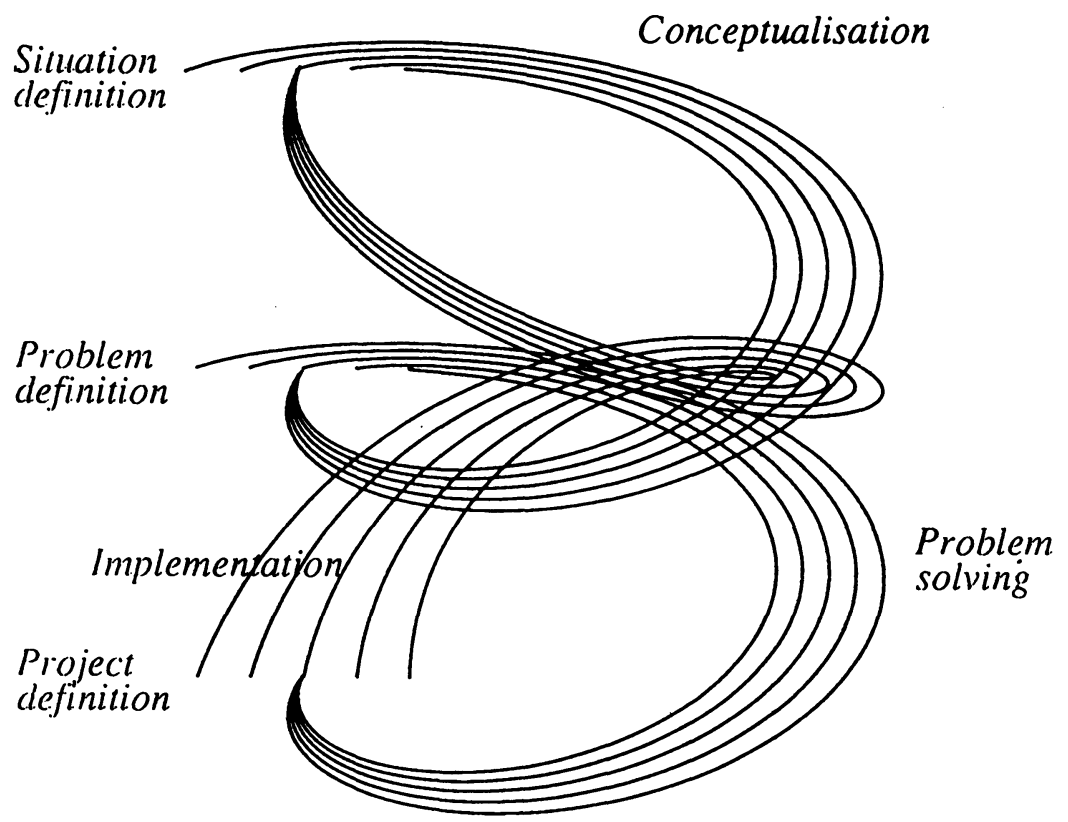

Figure 6 Situation, problem and implementation definition cycles 
On the other hand, this intrinsic need for "multi-levelness" of the presentation may be an answer to another conceptual difficulty with the existing methodologies for problem solving and decision making. I refer here to the fact that problem solving and decision making offer an obviously discrete-time vision of the world according to which both the problem and the choice make the time freeze, as it were, and disrupt the natural continuous flow of events. In one sense, this halting nature of problem solving or decision making is highly significant as it indicates that the time is ripe for posing, contemplation and rethinking. But, by the same token, it disrupts all the natural ties that exist between the problem and the processes that have led to its creation and cut it out of the natural texture of life. The line of thought I am advocating makes the future much less visible and provides for the connection between the events that have led to the perception of the problem to be explicitly accounted for.

These considerations of various facets of the temporal aspects of the presented framework seem to be a nice and fitting point to wind up the present discussion. Some of the multi-layer representations developed in the course of thinking out the way of solving some particular problem may turn out to be robust and persistent enough to be applicable for many other problems to come. In this case, they give rise to a new theory of an appropriate scale, while the reasons underlying its creation become almost immaterial as the theory progresses to its full maturity and while the corresponding research activity ceases to belong to the systems realm. Unfortunately (or, possibly, fortunately), most of the systems designed in this manner cannot claim the same robustness and long-term nature. They remain valid only for a limited period of time and, also, for rather unique circumstances.

It would be also useful to note that the multi-layer representation construed here comprises not only what has been retained in it explicitly but also what has been discarded. In this sense, all the rest of the systems analysis protocol should be kept at the back of our mind and be constantly used for systems monitoring, i.e., for checking the validity of the provisionally adopted vision against the new influx of evidence in terms of both what has been adopted and what has been discarded.

The same arguments should be applied to the theory at hand. Of course, I would be very pleased to see it as an enduring framework. But I am equally aware of the fact that it is very much preconditioned by the past developments in systems thinking and that its implementational depth is not yet as deep as one might desire. Therefore, it would be rather premature of me to forecast its viability and generality. But, even if its life-span is doomed to be short lived, I do hope that it may be a source of some new insights and as another option to be kept in mind and to provide a reference to check against in further developments of systems methodology. 


\section{ACKNOWLEDGEMENTS}

I feel impelled to express my gratitude to Dr. V. Antonuk, Academician S. Emelyanov, Academician J. Gvishiani, Dr. P. Humphreys, Dr. E. Mirskii and Dr. D. Pospelov, with whom I have had the opportunity to discuss these matters at length and whose comments were to me of great help and inspiration. I would also like to thank D. Tsoubelis for his work in developing the mathematical graphics programs which drew figures 5 and 6.

\section{REFERENCES}

Ackoff, R. L. and Emery, F. (1974) On Purposeful Systems. Aldine-Atherton, Chicago.

Adams, J. L. (1979) Conceptual Blockbusting. Norton, New York.

Berkeley, D. and Humphreys, P. C. (1982) Structuring Decision Problems and the "bias heuristic". Acta Psychologica, 50, 201-252.

Bobrow, D. G. and Collins, A. (1968) Representation avid Understanding: Studies in Cognitive Science. Academic Press, New York:.

Boden, M. (1977) Artificial Intelligence and Natural Man. Basic Books, New York.

de Bono, E. (1970) Lateral Thinking: A Textbook of Creativity. Wand Lock, London.

Checkland, P. (1981) Systems Thinking, Systems Practice. Wiley, Chichester.

Emelyanov, S. V. and Nappelbaum, E. L. (1977) Logic of Rational Choice, in: The State-of-the-Art Report in Science and Technology. Engineering Cybernetics. VINITI Publications, Moscow. (in Russian).

Emelyanov, S. V. and Nappelbaum, E. L. (1985) Systems, Purposefulness and Cognition, in Systems Research II.. Methodological Problems (ed. J. M. Gvishiani). Pergamon Press, Oxford.

Festinger, L. A. (1957) A Theory of Cognitive Dissonance. Row \& Peterson, Evanston.

Fodor, J. A. (1981) Representations. MIT Press, Cambridge, Mass.

van Gigch, J. P. (1987) Decision Making about Decision Making. Abacus Press, Cambridge, Mass.

Gordon, W. J. (1961) Synectics. Harper and Row, New York.

Heider, F. (1946) Attitudes and Cognitive Organization. Journal of Psychology, 21, 107-112.

Humphreys, P.C. and Berkeley , D. (1983). Problem Structuring Calculi and Levels of Knowledge Representation in Decision Making, in Decision Making under Uncertainty (ed. R. W. Scholz) North Holland, Amsterdam.

Humphreys, P.C. and Berkeley, D. (1985) Handling uncertainty: levels of analysis of decision problems, in Behavioural Decision Making: Theory and Analysis. (ed. G. N. Wright ) Plenum, New York. 
Jeffreys, R. (1965) The Logic of Decisions. McGraw Hill, New York.

Koberg, D. and Bagnall, J (1974) The Universal Traveller. A Soft-Systems Guidebook to Creativity, Problem-Solving and the Process of Design. William Kaufman, Los Altos, California.

Krantz, D. H., Luce, R. D., Suppes, P. and Tversky, A. (1971) Foundations of Measurement: Additive and Polynomial Representations. Academic Press, New York.

Lakatos, I. (1963-4) Proofs and Refutations. British Journal of Philosophy (in four parts).

Lancaster, K. J. (1966) A New Approach to Consumer Theory. Journal of Political Economy, 74 (1).

Larichev, 0.1. (1979) Science and Art of Decision Making. Nauka, Moscow. (in Russian).

Mandler, J. M. and Mandler, G. (1964) Thinking: From Association to Gestalt. Wiley, New York.

Maryuama, M. (1963) Generating Complex Patterns by Means of Simple Rules of Interaction. Methods, 14,17-26.

Mason, R. and Mitroff, I. (1981) Challenging Strategic Planning Assumptions. Wiley, New York.

Maturana, H. and Varela, F. (1979) Autopoiesis and Cognition. Riedel, Boston.

Minsky, M. (1975) A Framework for Representing Knowledge, in The Psychology of Computer Vision (ed. P. H. Winston) McGraw Hill, New York.

Newell, A. and Simon, H. A. (1972) Human Problem Solving. Prentice Hall, Englewood Cliffs.

Pask, 0. (1975) Conversation, Cognition and Learning. Elsevier, Amsterdam.

Pospelov, D. A. and Pushkin, V. N. (1975). Intelligence and Automata. Soviet Radio, Moscow. (in Russian).

Pospelov, D. A. (1981) Logic-Linguistic Models in Control Systems. Energoizdat, Moscow. (in Russian).

Schank, R. C. (1986) Explanation Patterns. Erlbaum, Hillsdale, N. J.

Simon, H. A. (1960) The New Science of Management Decisions. Harper and Row, New York.

Simon, H. A. (1967) Motivational and Emotional Control of Cognition. Psychological Review, 74, 29-39.

Simon, H. A. (1969) The Science of Artificial. MIT Press, Cambridge, Mass.

White, D. J. (1975) Decision Methodology. Wiley, Chichester. 


\section{BIOGRAPHY}

Eric L. Nappelbaum is Vice Chancellor at the Moscow School for Business of Political Science and Law and Research Director at the Moscow Institute of System Analysis, faculty of Operational Research. He studied Physics and Computer Sciences at the Moscow State University and did his PhD on Control Problems at the Moscow Physical Technical Institute. Dr Eric L. Nappelbaum is member of All Russian Academy of Science, Department of Operational Research and IT Strategy. Dr Eric Nappelbaum has published more than 40 international papers. He is Visiting Professor at the London School of Economics and Political Science and at the International Management Centre in Budapest. The research interests of $\mathrm{Dr}$ Nappelbaum include Organisational Transformation, System Analysis and System Logic. 